\title{
New Vector Description of Kinetic Pressures on Shaft Bearings of a Rigid Body Nonlinear Dynamics with Coupled Rotations around No Intersecting Axes
}

\author{
Katica R. (Stevanović) Hedrih*, Ljljana Veljović** \\ *Mathematical Institute SANU, Belgrade, Serbia, and Faculty of Mechanical \\ Engineering University of Niš, Serbia, E-mail: khedrih@eunet.rs \\ ** Faculty of Mechanical Engineering University of Kragujevac, Serbia, E-mail: \\ khedrih@sbb.rs
}

\begin{abstract}
New vector description of kinetic pressures on shaft bearings of a rigid body nonlinear dynamics with coupled rotations around no intersecting axes is first main result presented in this paper. Mass moment vectors and vector rotators coupled for pole and oriented axes, defined by K. Hedrih in 1991, are used for obtaining vector expressions for kinetic pressures on the shaft bearings of a rigid body dynamics with coupled rotations around no intersecting axes. A complete analysis of obtained vector expressions for kinetic pressures on shaft bearings give us a series of the kinematical vectors rotators around both directions determined by axes of the rigid body coupled rotations around no intersecting axes. As an example of defined dynamics, we take into consideration a heavy gyro-rotordisk with one degree of freedom and coupled rotations when one component of rotation is programmed by constant angular velocity. For this system with nonlinear dynamics, series of graphical presentation transformations in realizations with changes of eccentricity and angle of inclination (skew position) of heavy rigid body-disk in relation to self rotation axis are presented, as well as in realization with changing orthogonal distance between axes of coupled rotations. Angular velocity of kinetic pressures components in vector form are expressed by using angular acceleration and angular velocity of component coupled rotations of gyrorotor-disk.
\end{abstract}

Keywords: coupled rotation; no intersecting axes; deviational mass moment vector; rotator; kinetic pressures; kinetic pressure components; nonlinear dynamics; gyrorotordisk; eccentricity; angle of inclination, deviation kinetic couple; fixed point; graphical presentations; three parameter analysis 


\section{Introduction}

No precisions and errors in the functions of gyroscopes caused by eccentricity and unbalanced gyro rotor body as well distance between axes of rotations are reason to investigate determined task as in the title of our paper.

The classical book [1] by Andonov, Vitt and Haykin contain a classical and very important elementary dynamical model of heavy mass particle relative motion along circle around vertical axis through it's center. Nonlinear dynamics and singularities lead to primitive model of the simple case of the gyro-rotor, which represent an useful dynamical and mathematical model of nonlinear dynamics.

Using K. Hedrih's (See Refs. [2-11]) mass moment vectors and vector rotators, some characteristics vector expressions of linear momentum and angular momentum and their derivatives for rigid body single rotation, were obtain physical and dynamical visible properties of the complex system dynamics and their kinetic parameters in vector form for single rotation. There are vector components of the shaft bearing kinetic pressures with opposite directions and same intensity that present deviational couple effect containing vector rotators, whose directions are same as kinetic pressure components on corresponding rotor shaft bearings (for detail see Refs. [2] and [5]).

The definitions of mass moment vectors coupled to the pole and the axis [2-9], [12] are introduced in the foundation of this vector method. The main vector is $\overrightarrow{\mathrm{J}}_{\vec{n}}^{(O)} \stackrel{\text { def }}{=} \iiint_{V}[\vec{\rho},[\vec{n}, \vec{\rho}] d m$ of the body mass inertia moment at the point $A=O$ for the axis oriented by the unit vector and there is a corresponding vector $\vec{D}_{\vec{n}}^{(O)}$ of the rigid body mass deviational moment for the axis through the point (see References [2] and [5-6]).

This vector approach is very suitable to obtain new view to the properties of dynamics of pure classical system dynamics investigated by numerous generations of the researchers and serious scientists around the world. We proof this approach in our published reference [12]. In Introduction of this paper [12] a short reviews of the basis of the subjective selected references about original research results of dynamics and stability of gyrostats was given. Then is reason that we didn't made any reviews of the papers about gyroscopes.

Passing through the content of the numerous published scientific paper, we can see that no results concerning behavior of the kinetic pressure directions and intensity depending of the nonlinear dynamic regimes. Then, our aim is to investigate kinetic pressures and deviation kinetic couple appearing to the shaft bearings of the rigid body coupled rotations around two no intersecting axes. Two our References [12] from (2008 and 2010) contain short presentation of the kinetic 
pressure to the gyrorotor self rotation bearings and rotators, as well as presentation of the nonlinear dynamics of the heavy gyro-rotor, but not completed.

This is reason that we take into a large consisderation and investigation three parameters analysis of the vector expressions of shaft bearing kinetic presures and their cmponents based on our previous results on applications vector method and published in our References [12]. This paper contan new rezults based on the previous our results.

Organizations of this paper based on the vector method applications with use of the mass moment vectors and vector rotators for describing vector expressions of kinetic pressures of the shaft bearings, of the rigid body coupled rotations around two no intersecting axes and corresponding kinetic deviation couple appearing by opposite kinetic pressures to shaft bearings and shaft bearing reactions.

Dynamics of a gyro-rotor with one degree of freedom and coupled rotations when one component of rotation is programmed by constant angular velocity is considered, as an example. For this system of nonlinear dynamics, the series of graphical presentation of the kinetic pressures of the shaft bearing of a rigid body self rotation are presented.

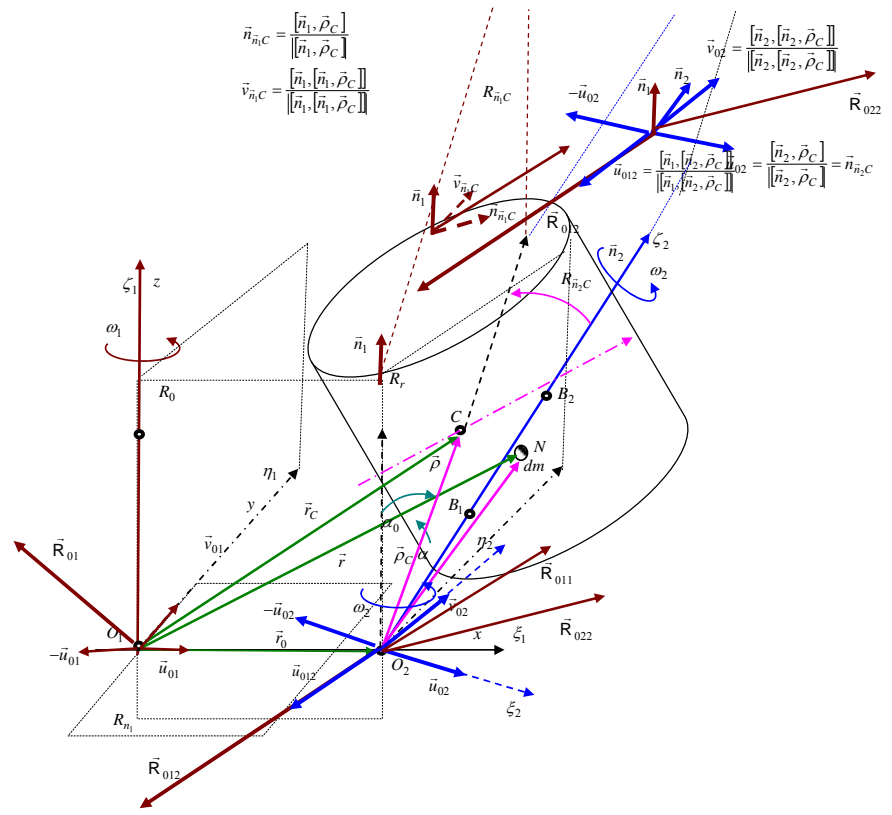

Figure 1

A rigid body coupled rotations around two no intersecting axes. System is with two degrees of mobility and one degrees of freedom, where $\varphi_{1}$ and $\varphi_{2}$ are rheonomic and generalized coordinates. Vector rotators $\vec{R}_{01}, \vec{R}_{011}$ and $\vec{R}_{022}$ are presented.ssential connections 


\section{Model of a Rigid Body Rotation Around Two Axes without Intersection}

Let us to consider rigid body coupled rotations around two no intersecting axes, presented in Figure 1. Ffirst axis is oriented by unit vector $\vec{n}_{1}$ with fixed position and second axis is oriented by unit vector $\vec{n}_{2}$ which is rotating around fixed axis with angular velocity $\vec{\omega}_{1}=\omega_{1} \vec{n}_{1}$. Axes of rotation are no intersecting axes. Rigid body is positioned on the moving rotating axis oriented by unit vector $\vec{n}_{2}$ and rotate around self rotating axis with angular velocity $\vec{\omega}_{2}=\omega_{2} \vec{n}_{2}$ and around fixed axis oriented by unit vector $\vec{n}_{1}$ with angular velocity $\vec{\omega}_{1}=\omega_{1} \vec{n}_{1}$. All geometrical parameters are presented in Fgure 1.

When any of three main central axes of rigid body mass inertia moments is not in direction of self rotation axis, then we can see that rigid body is scew positioned to the body self rotation axis. The angles of rigid body central main inertia axes inclinations acording self-lf rotation axis are $\beta_{i}, i=1,2$. These angles are angles of scew position of rigid body to the body self rotation axis. When center $C$ of the mass of rigid body is not on body self rotation axis of rigid body rotation, we can say that rigid body is scew positioned. Eccentricity of body position is normal distance between body mass center $C$ and axis of self rotation and it is defined by $\vec{e}=\left[\vec{n}_{2},\left[\vec{\rho}_{C}, \vec{n}_{2}\right]\right]$. Here $\vec{\rho}_{C}$ is vector position of mass center $C$ with origin in point $\mathrm{O}_{2}$, and position vector of mass center with fixed origin in point $\mathrm{O}_{1}$ is $\vec{r}_{C}=\vec{r}_{O}+\vec{\rho}_{C}$.

\section{Vector Equations of Dynamic Equlibrium of Rigid Body Coupled Rotations around Two No Intersecting Axes}

By using theorems of linear momentum and angular momentum with respect to time, we can write two equations of dynamic equilibrium of the considered rigid body coupled rotations about two no intersecting axes, presented in Figure 1, in the following equations (for detail see Ref. [17] and Appendix):

$$
\begin{aligned}
\frac{d \overrightarrow{\mathrm{K}}}{d t} & =\overrightarrow{\mathrm{R}}_{01}\left|\left[\vec{n}_{1}, \vec{r}_{0}\right]\right| M+\overrightarrow{\mathrm{R}}_{011}\left|\overrightarrow{\mathrm{S}}_{\vec{n}_{1}}^{\left(O_{2}\right)}\right|+\overrightarrow{\mathrm{R}}_{022}\left|\overrightarrow{\mathrm{S}}_{\vec{n}_{2}}^{\left(O_{2}\right)}\right|+2 \omega_{1} \omega_{2}\left[\vec{n}_{1}, \overrightarrow{\mathrm{S}}_{\vec{n}_{2}}^{\left(O_{2}\right)}\right]= \\
& =\vec{G}+\vec{F}_{A N 1}+\vec{F}_{B N 1}+\vec{F}_{A m}+\sum_{i=1}^{i=P} \vec{F}_{i}=\vec{G}+\vec{F}_{A N 2}+\vec{F}_{B N 2}+\vec{F}_{A m 2}+\sum_{i=1}^{i=P} \vec{F}_{i}
\end{aligned}
$$




$$
\begin{aligned}
\frac{d \overrightarrow{\mathrm{L}}_{O_{1}}}{d t} & =\vec{\chi}_{12}\left(\vec{r}_{0}, \vec{\rho}_{C}, M, \dot{\omega}_{1}, \dot{\omega}_{2}, \omega_{1}, \omega_{2}, \vec{n}_{1}, \vec{n}_{2}\right)+\dot{\omega}_{1} \vec{n}_{1} r_{0}^{2} M+2 \omega_{1} \omega_{2}\left[\vec{n}_{1}, \overrightarrow{\mathrm{J}}_{\bar{n}_{2}}^{\left(O_{2}\right)}\right] \\
& +\dot{\omega}_{1}\left(\vec{n}_{1}, \overrightarrow{\mathrm{J}}_{\vec{n}_{1}}^{\left(O_{2}\right)},\right) \vec{n}_{1}+\dot{\omega}_{2}\left(\vec{n}_{2}, \overrightarrow{\mathrm{J}}_{\vec{n}_{2}}^{\left(O_{2}\right)},\right) \vec{n}_{2}+\overrightarrow{\mathrm{R}}_{1}\left|\overrightarrow{\mathrm{D}}_{\vec{n}_{1}}^{\left(O_{2}\right)}\right|+\overrightarrow{\mathrm{R}}_{2}\left|\overrightarrow{\mathrm{D}}_{\vec{n}_{2}}^{\left(O_{2}\right)}\right|= \\
& =\left[\vec{r}_{0}+\vec{\rho}_{C}, \vec{G}\right]+\left[\vec{\rho}_{B 1}, \vec{F}_{B N 1}\right]+\sum_{i=1}^{i=P}\left[\vec{r}_{0}+\vec{\rho}_{i,}, \vec{F}_{i}\right]=\left[\vec{r}_{0}+\vec{\rho}_{C}, \vec{G}\right]+ \\
& +\left[\vec{r}_{0}+\vec{\rho}_{A 2}, \vec{F}_{A N 2}\right]+\left[\vec{r}_{0}+\vec{\rho}_{B 2}, \vec{F}_{B N 2}\right]+\left[\vec{r}_{0}+\vec{\rho}_{A 2}, \vec{F}_{A m 2}\right]+\sum_{i=1}^{i=P}\left[\vec{r}_{0}+\vec{\rho}_{i,}, \vec{F}_{i}\right]
\end{aligned}
$$

where $\vec{F}_{i}, i=1,2,3 \ldots, P$ are active forces and $\vec{G}$ is weight of gyro rotor, $\vec{F}_{A 1}$ and $\vec{F}_{B 1}$ are reactive forces of fixed axis shaft bearing reactions and $\vec{F}_{A 2}$ and $\vec{F}_{B 2}$ are forces of self rotation shaft bearing reactions. From previous obtained vector equations, it is not difficult to obtain kinetic pressures to both shaft bearings, $\vec{F}_{A 1}$ and $\vec{F}_{B 1}$, as well as $\vec{F}_{A 2}$ and $\vec{F}_{B 2}$ on both shafts bearings as well as two differential equations along $\varphi_{1}$ and $\varphi_{2}$ of the rigid body coupled rotations about two no intersecting axes, and to obtain time solutions of unknown generalized coordinate $\varphi_{1}$ and $\varphi_{2}$, or if we know these coordinate to find unknown external active forces.

For the case that axes are perpendicular some terms in previous vector expressions and vector equations are equal to zero, but these equations are nonlinear along angle coordinates $\varphi_{1}$ and $\varphi_{2}$, and coupled by generalized coordinates, $\varphi_{1}$ and $\varphi_{2}$, and their derivatives, and also, by forces of shaft bearings reactions.

Two vector equates (1) and (2) are valid for rigid body coupled rotations around no intersecting axes, as well for the case intersecting axes as its special case. Also, these equations are valid for the system dynamics with two degrees of mobility, and for three different cases.

\section{Vector Rotators of Rigid Body Coupled Rotations around Two No Intersecting Axes}

We can see that in previous vector equations (1) and (2) terms for derivative of linear momentum and angular momentum contain two sets of the vector rotators:

$$
\begin{aligned}
& \overrightarrow{\mathrm{R}}_{01}=\dot{\omega}_{1}\left[\vec{n}_{1}, \frac{\vec{r}_{0}}{r_{0}}\right]+\omega_{1}^{2}\left[\vec{n}_{1},\left[\vec{n}_{1}, \frac{\vec{r}_{0}}{r_{0}}\right]\right], \quad \overrightarrow{\mathrm{R}}_{011}=\dot{\omega}_{1} \frac{\overrightarrow{\mathrm{S}}_{\vec{n}_{1}}^{\left(O_{2}\right)}}{\left|\overrightarrow{\mathrm{S}}_{\bar{n}_{1}}^{\left(O_{2}\right)}\right|}+\omega_{1}^{2}\left[\vec{n}_{1}, \frac{\overrightarrow{\mathrm{S}}_{\vec{n}_{1}}^{\left(O_{2}\right)}}{\left|\overrightarrow{\mathrm{S}}_{\vec{n}_{1}}^{\left(O_{2}\right)}\right|}\right] \\
& \left|\overrightarrow{\mathrm{R}}_{012}\right|=\mathrm{R}_{012}=2 \omega_{1} \omega_{2}=2 \omega_{1} \omega_{2}
\end{aligned}
$$




$$
\overrightarrow{\mathrm{R}}_{022}=\dot{\omega}_{2} \frac{\overrightarrow{\mathrm{S}}_{\bar{n}_{2}}^{\left(O_{2}\right)}}{\left|\overrightarrow{\mathrm{S}}_{\bar{n}_{2}}^{\left(O_{2}\right)}\right|}+\omega_{2}^{2}\left[\vec{n}_{2}, \frac{\overrightarrow{\mathrm{S}}_{\bar{n}_{2}}^{\left(O_{2}\right)}}{\left|\overrightarrow{\mathrm{S}}_{\bar{n}_{2}}^{\left(O_{2}\right)}\right|}\right], \quad \overrightarrow{\mathrm{R}}_{012}=2 \omega_{1} \omega_{2} \frac{\left|\vec{n}_{1}, \overrightarrow{\mathrm{S}}_{\vec{n}_{2}}^{\left(O_{2}\right)}\right|}{\left|\vec{n}_{1}, \overrightarrow{\mathrm{S}}_{\bar{n}_{2}}^{\left(O_{2}\right)}\right|}
$$

First two vector rotators $\vec{R}_{01}$ and $\vec{R}_{011}$ are orthogonal to the direction of the first fixed axis and third vector rotator is orthogonal to the self rotation axis. But, first vector rotator $\overrightarrow{\mathrm{R}}_{01}$ is coupled for pole $O_{1}$ on the fixed axis and second and third vector rotators, $\vec{R}_{011}$ and $\vec{R}_{022}$, are coupled for the pole $O_{2}$ on self rotation axis and for corresponding direction oriented by directions of component angular velocities of coupled rotations. Intensities of two first rotators are equal and are expressed by angular velocity and angular acceleration of the first component rotation, and intensity of third vector rotators is expressed by angular velocity and angular acceleration of the second component rotation, and they are in the following forms: $\mathrm{R}_{01}=\mathrm{R}_{011}=\sqrt{\dot{\omega}_{1}^{2}+\omega_{1}^{4}} \quad$ and $\quad \mathrm{R}_{022}=\sqrt{\dot{\omega}_{2}^{2}+\omega_{2}^{4}}$.

Lets introduce notation $\gamma_{01}, \gamma_{011}$ and $\gamma_{022}$ denote difference between corresponding component angles of rotation $\varphi_{1}$ and $\varphi_{2}$ of the rigid body component rotations and corresponding absolute angles of rotation of pure kinematics vector rotators about axes oriented by unit vectors $\vec{n}_{1}$ and $\vec{n}_{2}$. These angular velocities of relative kinematics vectors rotators $\vec{R}_{01}, \vec{R}_{011}$ and $\vec{R}_{022}$ which rotate about corresponding axis in relation to the component angular velocities of the rigid body component rotations are:

$$
\dot{\gamma}_{01}=\dot{\gamma}_{011}=\frac{\dot{\varphi}_{1}\left(2 \ddot{\varphi}_{1}-\dot{\varphi}_{1} \dddot{\varphi}_{1}\right)}{\ddot{\varphi}_{1}^{2}+\dot{\varphi}_{1}^{4}} \quad \text { and } \quad \dot{\gamma}_{02}=\frac{\dot{\varphi}_{2}\left(2 \ddot{\varphi}_{2}-\dot{\varphi}_{2} \dddot{\varphi}_{2}\right)}{\ddot{\varphi}_{2}^{2}+\dot{\varphi}_{2}^{4}}
$$

In Figure 1 Vector rotators $\vec{R}_{01}, \vec{R}_{011}$ and $\vec{R}_{022}$ are presented.

We can see that in previous vector expression (2) for derivative of angular momentum are introduced vector rotators in the following vector form:

$$
\begin{aligned}
& \overrightarrow{\mathrm{R}}_{1}=\dot{\omega}_{1} \frac{\overrightarrow{\mathrm{D}}_{\bar{n}_{1}}^{\left(O_{2}\right)}}{\left|\overrightarrow{\mathrm{D}}_{\bar{n}_{1}}^{\left(O_{O_{2}}\right)}\right|}+\omega_{1}^{2}\left[\vec{n}_{1}, \frac{\overrightarrow{\mathrm{D}}_{\bar{n}_{1}}^{\left(O_{2}\right)}}{\left|\overrightarrow{\mathrm{D}}_{\vec{n}_{1}}^{\left(O_{2}\right)}\right|}\right], \quad \overrightarrow{\mathrm{R}}_{2}=\dot{\omega}_{2} \frac{\overrightarrow{\mathrm{D}}_{\vec{n}_{2}}^{\left(O_{2}\right)}}{\left|\overrightarrow{\mathrm{D}}_{\vec{n}_{2}}^{\left(O_{2}\right)}\right|}+\omega_{2}^{2}\left[\vec{n}_{2}, \frac{\overrightarrow{\mathrm{D}}_{\vec{n}_{2}}^{\left(O_{2}\right)}}{\left|\overrightarrow{\mathrm{D}}_{\vec{n}_{2}}^{\left(O_{2}\right)}\right|}\right] \\
& \overrightarrow{\mathrm{R}}_{12}=2 \omega_{1} \omega_{2} \underset{\left.\vec{n}_{1}, \overrightarrow{\mathrm{J}}_{\vec{n}_{2}}^{\left(O_{2}\right)}\right]}{\left|\vec{n}_{1}, \overrightarrow{\mathrm{J}}_{\vec{n}_{2}}^{\left(O_{2}\right)}\right|}=2 \omega_{1} \omega_{2} \vec{u}_{12}
\end{aligned}
$$




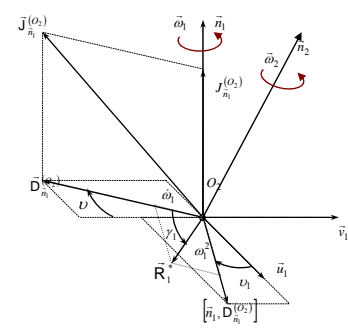

$a^{*}$

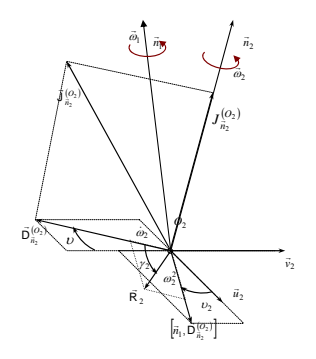

$b^{*}$

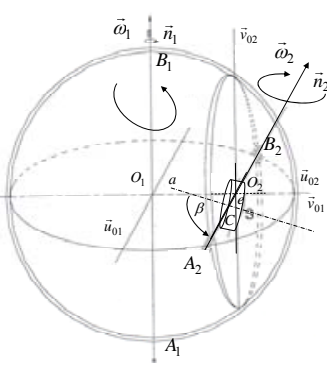

$c^{*}$

Figure 2

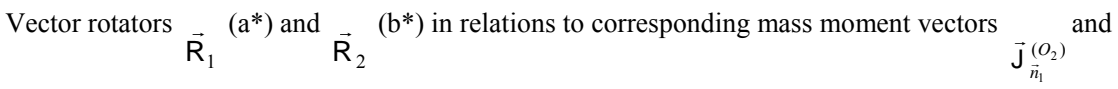

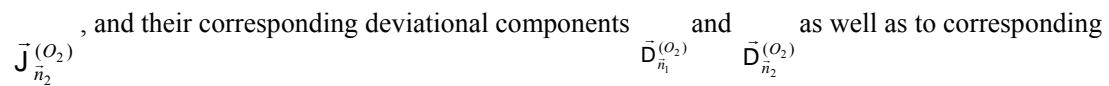
deviational planes. ( $\left.\mathrm{c}^{*}\right)$ Model of heavy gyro rotor with two component coupled rotations around orthogonal axes without intersections

The first $\vec{R}_{1}$ is orthogonal to the fixed axis oriented by unit vector $\vec{n}_{1}$ and second $\vec{R}_{2}$ is orthogonal to the self rotation axis oriented by unit vector $\vec{n}_{2}$. Intensity of first rotator $\vec{R}_{1}$ is equal to intensity of previous defined rotator $R_{01}$ and intensity of second rotator $\vec{R}_{2}$ is equal to intensity of previous defined rotator $R_{022}$ defined by expressions (7). In Figure 2 vector rotators $\vec{R}_{1}$ (in Figure $2 a^{*}$ ) and $\vec{R}_{2}$ (in Figure 2.b*) in relations to corresponding mass moment vectors $\vec{j}_{\vec{n}_{1}}\left(\mathrm{O}_{2}\right)$ and $\overrightarrow{\mathrm{j}}{\overrightarrow{n_{2}}}_{2}^{\left(O_{2}\right)}$, and their corresponding deviational components $\overrightarrow{\mathrm{D}}_{\vec{n}_{1}}^{\left(\mathrm{O}_{2}\right)}$ and $\overrightarrow{\mathrm{D}}_{\vec{n}_{2}}^{\left(O_{2}\right)}$ as well as to corresponding deviational planes are presented. Vector rotators $\vec{R}_{1}$ and $\vec{R}_{2}$ are pure kinematical vectors first presented in reference $[18,19]$ as a function on angular velocity and angular accelerationin a form $\vec{R}=\ddot{\varphi} \vec{u}+\dot{\varphi}^{2} \vec{w}=R \vec{R}_{0}$.

Rotators from first set are rotated around through pole $\mathrm{O}_{2}$ and axis in direction of first component rotation angular velocity and depend of angular velocity $\omega_{1}$ and angular acceleration $\dot{\omega}_{1}$. There are two vectors of such type and all trees have equal intensity. Rotators from second set are rotated around axis in direction of second component rotation and depend of angular velocity $\omega_{2}$ and angular acceleration $\dot{\omega}_{2}$. There are two vectors of such type and they have equal intensity. 
Lets introduce notation $\gamma_{1}$, and $\gamma_{2}$ denote difference between corresponding component angles of rotation $\varphi_{1}$ and $\varphi_{2}$ of the rigid body component rotations and corresponding absolute angles of pure kinematics vector rotators about axes oriented by unit vectors $\vec{n}_{1}$ and $\vec{n}_{2}$ through pole $O_{2}$. These angular velocity of relative kinematics vectors rotators $\vec{R}_{1}$ and $\vec{R}_{2}$ which rotate about axes in corresponding directions in relation to the component angular velocities of the rigid body component rotations through pole $\mathrm{O}_{2}$ are expressed as $\dot{\gamma}_{1}=\dot{\gamma}_{01}=\dot{\gamma}_{011}$ and $\dot{\gamma}_{2}=\dot{\gamma}_{02}$.

\section{Vector Expressions of Kinetic Pressures (Kinetic Reactions) on Shaft Bearings of Rigid Body Coupled Rotations around Two No Intersecting Axes}

Kinetic pressures (bearing reactions with out parts reactions induced by external forces) on fixed shaft bearings for the case that spherical bearing is at the pole $O_{1}$ and cylindrical in this fixed axis defined by vector position $\vec{\rho}_{B 1}=\rho_{B 1} \vec{n}_{1}$ are in the following form:

$$
\begin{aligned}
& \vec{F}_{A N 1}=-\vec{F}_{B N 1}+\vec{R}_{011}\left|\vec{S}_{\vec{n}_{1}}^{\left(O_{2}\right)}\right|+\vec{R}_{022}\left|\overrightarrow{\mathrm{S}}_{\vec{n}_{2}}^{\left(O_{2}\right)}\right|+2 \omega_{1} \omega_{2}\left[\vec{n}_{1}, \overrightarrow{\mathrm{S}}_{\vec{n}_{2}}^{\left(O_{2}\right)}\right] \\
& \vec{F}_{B N 1}=\frac{1}{\rho_{B 1}}\left[\vec{R}_{1}, \vec{n}_{1}\right]\left|\overrightarrow{\mathrm{D}}_{\vec{n}_{1}}^{\left(O_{2}\right)}\right|+\frac{1}{\rho_{B}}\left[\overrightarrow{\mathrm{R}}_{2}, \vec{n}_{1,}\right] \overrightarrow{\mathrm{D}}_{\left.\vec{n}_{2}\right)}^{\left(O_{2}\right)} \mid+\frac{2 \omega_{1} \omega_{2}}{\rho_{B}} \llbracket \vec{n}_{1}, \overrightarrow{\mathrm{J}} \vec{n}_{2}\left(O_{2}\right), \vec{n}_{1,} \rrbracket \\
& +\frac{1}{\rho_{B 1}}\left[\vec{\chi}_{12}\left(\vec{r}_{0}, \vec{\rho}_{C}, M, \dot{\omega}_{1}, \dot{\omega}_{2}, \omega_{1}, \omega_{2}, \vec{n}_{1}, \vec{n}_{2}\right), \vec{n}_{1,}\right]+\frac{\dot{\omega}_{2}}{\rho_{B}}\left(\vec{n}_{2}, \overrightarrow{\mathrm{J}}_{\vec{n}_{2}}^{\left(O_{2}\right)},\left[\vec{n}_{2}, \vec{n}_{1}\right]=-\vec{F}_{B N 1}^{d e v}\right.
\end{aligned}
$$

It is not difficult, by use system decomposition, to obtain kinetic pressures on body self rotation shaft bearings for the case that spherical bearing is at the pole $\mathrm{O}_{2}$.

By analysis vector equations (1) and (2) and corresponding expressions (8) and (9) for kinetic pressures on the both shafts bearings, we can conclude that in the system to the both shaft bearings appear in the pair of bearings two opposite components of kinetic pressures with deviation couple. In fixed shaft bearings $A_{1}$ and $B_{1}$ appear the following opposite components: $\vec{F}_{B N 1}$ and $\vec{F}_{B N 1}^{d e v}$ in vector relation: $\vec{F}_{B N 1}=-\vec{F}_{B N 1}^{d e v}$, but in different points of appearance, bearings $A_{1}$ and $B_{1}$ with distance $\vec{\rho}_{B 1}$ and build one couple, $\overrightarrow{\mathrm{M}}_{\operatorname{dev} 1}=\left[\vec{\rho}_{B 1}, \vec{F}_{B N 1}\right]=-\left[\vec{\rho}_{B 1}, \vec{F}_{A N 1}^{d e v}\right]$, 
known under the name deviation couple, and identified in like our investigated system dynamics, for which we obtain the following vector expression:

$$
\begin{aligned}
\overrightarrow{\mathrm{M}}_{\operatorname{dev} 1}= & \overrightarrow{\mathrm{R}}_{1}\left|\overrightarrow{\mathrm{D}}_{\vec{n}_{1}}^{\left(O_{2}\right)}\right|+\left[\vec{n}_{1},\left[\overrightarrow{\mathrm{R}}_{2}, \vec{n}_{1} \rrbracket\left|\overrightarrow{\mathrm{D}}_{\vec{n}_{2}}^{\left(O_{2}\right)}\right|+2 \omega_{1} \omega_{2}\left[\vec{n}_{1}, \overrightarrow{\mathrm{J}}_{\vec{n}_{2}}^{\left(O_{2}\right)}\right]+\right.\right. \\
& +\left[\vec{n}_{1},\left[\vec{\chi}_{12}\left(\vec{r}_{0}, \vec{\rho}_{C}, M, \dot{\omega}_{1}, \dot{\omega}_{2}, \omega_{1}, \omega_{2}, \vec{n}_{1}, \vec{n}_{2}\right), \vec{n}_{1}\right]\right]
\end{aligned}
$$

Also, it is possible to conclude for two opposite components of kinetic pressures to the self rotation shaft bearings $\vec{F}_{B N 2}$ and $\vec{F}_{2}^{d e v}$ in vector relation: $\vec{F}_{B N 2}=-\vec{F}_{B N 2}^{d e v}$, but in different points of appearance, bearings $A_{2}$ and $B_{2}$ with distance $\vec{\rho}_{B 2}$ and build one couple, $\vec{M}_{d e v 2}=\left[\vec{\rho}_{B 2}, \vec{F}_{B N 2}\right]=-\left[\vec{\rho}_{B 2}, \vec{F}_{A N 2}^{d e v}\right]$, known under the name deviation couple, and also identified in like our investigated system dynamics.

\section{Dynamic of Rigid Body Coupled Rotations around Two Orthogonal No Intersecting Axes and with One Degree of Freedom}

We are going to take into consideration special case of the considered heavy rigid body with coupled rotations about two axes without intersection with one degree of freedom, and in the gravitation field. For this case generalized coordinate $\varphi_{2}$ is independent, and coordinate $\varphi_{1}$ is programmed. In that case, we say that coordinate $\varphi_{1}$ is rheonomic coordinate and system is with kinematical excitation, programmed by forced support rotation by constant angular velocity. When the angular velocity of shaft support axis is constant, $\dot{\varphi}_{1}=\omega_{1}=$ const, we have that rheonomic coordinate is linear function of time, $\varphi_{1}=\omega_{1} t+\varphi_{10}$, and angular acceleration around fixed axis is equal to zero $\dot{\omega}_{1}=0$. Special case is when the support shaft axis is vertical and the gyro-rotor shaft axis is horizontal, and all time in horizontal plane, and when axes are no intersecting at normal distance $a$. So we are going to consider that example presented in Figure $2 c^{*}$. The normal distance between axes is $a$. The angle of self rotation around moveable self rotation axis oriented by the unit vector $\vec{n}_{2}$ is $\varphi_{2}$ and the angular velocity is $\omega_{2}=\dot{\varphi}_{2}$. The angle of rotation around the shaft support axis oriented by the unit vector $\vec{n}_{1}$ is $\varphi_{1}$ and the angular velocity is $\omega_{1}=$ constat . The angular velocity of rotor is $\vec{\omega}=\omega_{1} \vec{n}_{1}+\omega_{2} \vec{n}_{2}=\dot{\varphi}_{1} \vec{n}_{1}+\dot{\varphi}_{2} \vec{n}_{2}$. The angle $\varphi_{2}$ is generalized coordinates in case when, we investigate system with one degrees of freedom, but system have two degrees of mobility. Also, without loose of generality, we take that rigid body 
is a disk, eccentrically positioned on the self rotation shaft axis with eccentricity $e$, and that angle of skew inclined position between one of main axes of disk and self rotation axis is $\beta$, as it is visible in Figure $2 c^{*}$.

For that example, differential equation of the heavy gyro rotor-disk self rotation of reviewed model in Figure 2 for the case coupled rotations about two orthogonal no intersecting axes by using (2), after multiplying scalar by $\vec{n}_{2}$, and taking into account orthogonal between axes of coupled rotations, we can obtain in the following form:

$$
\ddot{\varphi}_{2}+\Omega^{2}\left(\lambda-\cos \varphi_{2}\right) \sin \varphi_{2}+\Omega^{2} \psi \cos \varphi_{2}=0
$$

where

$$
\Omega^{2}=\omega_{1}^{2} \frac{J_{\bar{u}_{2}}^{(C)}-J_{\bar{v}_{2}}^{(C)}}{J_{\bar{n}_{2}}^{(C)}}, \lambda=\frac{m g e \sin \beta}{\omega_{1}^{2}\left(J_{\bar{u}_{2}}^{(C)}-J_{\vec{v}_{2}}^{(C)}\right)}, \psi=\frac{2 m e a \sin \beta}{J_{\bar{u}_{2}}^{(C)}-J_{\dot{v}_{2}}^{(C)}}, \varepsilon=1+4\left(\frac{e}{r}\right)^{2}
$$

Here it is considered an eccentric disc (eccentricity is $e$ ), with mass $m$ and radius $r$, which is inclined to the axis of its own self rotation by the angle $\beta$ (see Figure 4), so that previous constants (12) in differential equation (11) become the following forms:

$$
\Omega^{2}=\omega_{1}^{2} \frac{\left(\varepsilon \sin ^{2} \beta-1\right)}{\left(\varepsilon \sin ^{2} \beta+1\right)}, \varepsilon=1+4\left(\frac{e}{r}\right)^{2}, \lambda=\frac{g(\varepsilon-1) \sin \beta}{e \omega_{1}^{2}\left(\varepsilon \sin ^{2} \beta-1\right)}, \psi=\frac{2 e a \sin \beta}{e r\left(\varepsilon \sin ^{2} \beta-1\right)}
$$

Relative nonlinear dynamics of the heavy gyro-rotor-disk around self rotation shaft axis is possible to present by means of phase portrait method. Forms of phase trajectories and their transformations by changes of initial conditions, and for different cases of disk eccentricity and angle of its skew position, as well as for different values of orthogonal distance between axes of component rotations may present character of nonlinear oscillations.

For that reason it is necessary to find first integral of the differential equation (11). After integration of the differential equation (26), the non-linear equation of the phase trajectories of the heavy gyro rotor disk dynamics with the initial conditions $t_{0}=0, \varphi_{1}\left(t_{0}\right)=\varphi_{10}, \dot{\varphi}_{1}\left(t_{0}\right)=\dot{\varphi}_{10}$, we obtain in the following form:

$$
\dot{\varphi}_{2}^{2}=\dot{\varphi}_{02}^{2}+2 \Omega^{2}\left(\lambda \cos \varphi_{2}-\frac{1}{2} \cos ^{2} \varphi_{2}+\psi \sin \varphi_{2}\right)-2 \Omega^{2}\left(\lambda \cos \varphi_{02}-\frac{1}{2} \cos ^{2} \varphi_{02}+\psi \sin \varphi_{02}\right)
$$

The analyzed system is conservative and equation (14) is the energy integral. 
In considered case for the heavy gyro-rotor-disk nonlinear dynamics in the gravitational field with one degree of freedom and with constant angular velocity about fixed axis, we have three sets of vector rotators.

Three of these vector rotators $\vec{R}_{01}, \vec{R}_{011}$ and $\vec{R}_{1}$, from first set, are with same constant intensity $\left|\vec{R}_{01}\right|=\left|\vec{R}_{011}\right|=\left|\vec{R}_{1}\right|=\omega_{1}^{2}=$ cons $\tan t$ and rotate with constant angular velocity $\omega_{1}$ and equal to the angular velocity of rigid body component precession rotation about fixed axis, but two of these three vector rotators, $\vec{R}_{011}$ and $\vec{R}_{1}$ are connected to the pole $O_{2}$ on the self rotation axis, and are orthogonal to the axis parallel direction as direction of the fixed axis. All these three vector rotators $\vec{R}_{01}, \vec{R}_{011}$ and $\vec{R}_{1}$ are in different directions (see Figures 1 and 3). Two of these vector rotators, $\vec{R}_{022}$ and $\vec{R}_{2}$, from second set, are with same intensity equal to $\mathrm{R}_{022}=\sqrt{\dot{\omega}_{2}^{2}+\omega_{2}^{4}}$, and connecter to the pole $\mathrm{O}_{2}$ and orthogonal to the self rotation axis oriented by unit vector $\vec{n}_{2}$ and rotate about this axis with relative angular velocity $\dot{\gamma}_{2}$ defined by expression (6), in respect to the self rotation angular velocity $\omega_{2}$ (see Figures $2 a^{*}, b^{*}$ and $c^{*}$ ).

By use expressions (3-5) and (7), we can list following series of vector rotators of the gyro-rotor-disk with coupled rotation around orthogonal no intersecting axes and with $\omega_{1}=$ const :

$$
\begin{aligned}
& \vec{R}_{01}=\omega_{1}^{2} \vec{v}_{01}, \quad\left|\overrightarrow{\mathrm{R}}_{01}\right|=\omega_{1}^{2}, \quad \overrightarrow{\mathrm{R}}_{011}=-\omega_{1}^{2} \frac{\left\langle\sin \widetilde{\beta} \sin \varphi_{2} \vec{v}_{01}+\cos \widetilde{\beta} \vec{u}_{01}\right\rangle}{\sqrt{\cos ^{2} \widetilde{\beta}+\sin ^{2} \widetilde{\beta} \sin ^{2} \varphi_{2}}},\left|\overrightarrow{\mathrm{R}}_{011}\right|=\omega_{1}^{2} \\
& \overrightarrow{\mathrm{R}}_{022}=\dot{\omega}_{2} \vec{v}_{02}-\omega_{1}^{2} \vec{u}_{02}, \quad\left|\overrightarrow{\mathrm{R}}_{022}\right|=\sqrt{\dot{\omega}_{2}^{2}+\omega_{2}^{4}}, \quad \overrightarrow{\mathrm{R}}_{012}=-2 \omega_{1} \omega_{2} \vec{u}_{01}, \\
& \left|\overrightarrow{\mathrm{R}}_{012}\right|=\mathrm{R}_{012}=2 \omega_{1} \omega_{2}, \quad \overrightarrow{\mathrm{R}}_{1}=-\omega_{1}^{2} \frac{\vec{u}_{01}\left\langle\sin \widetilde{\beta} \cos \varphi_{2}\right\rangle+\vec{v}_{01}\langle\cos \widetilde{\beta}\rangle}{\sqrt{\cos ^{2} \beta+\sin ^{2} \beta \cos ^{2} \varphi_{2}}}, \quad\left|\overrightarrow{\mathrm{R}}_{1}\right|=\omega_{1}^{2} \\
& \overrightarrow{\mathrm{R}}_{2}=\dot{\omega}_{2} \vec{v}_{02}-\omega_{2}^{2} \vec{u}_{02}, \quad\left|\overrightarrow{\mathrm{R}}_{2}\right|=\sqrt{\dot{\omega}_{2}^{2}+\omega_{2}^{4}}, \quad \overrightarrow{\mathrm{R}}_{12}=2 \omega_{1} \omega_{2} \mid \frac{\left|\vec{n}_{1}, \overrightarrow{\mathrm{J}}_{\vec{n}_{2}}^{\left(O_{2}\right)}\right|}{\left|\vec{n}_{1}, \overrightarrow{\mathrm{J}}_{\vec{n}_{2}}^{\left(O_{2}\right)}\right|}=2 \omega_{1} \omega_{2} \vec{u}_{12}, \\
& \left|\overrightarrow{\mathrm{R}}_{12}\right|=\mathrm{R}_{12}=2 \omega_{1} \omega_{2}
\end{aligned}
$$

in which $\widetilde{\beta}$ is angle between relative vector position $\vec{\rho}_{C}$ of rigid body mass center $C$ and self rotation axis oriented by unit vector $\vec{n}_{2}$. One of the vectors rotators from the third set is $\vec{R}_{012}$ with intensity $\left|\vec{R}_{012}\right|=2 \omega_{1} \omega_{2}$ and direction: 
$\vec{R}_{012}=-2 \omega_{1} \omega_{2} \vec{u}_{01}$. This vector rotator is connecter to the pole $O_{2}$ and orthogonal to the axis oriented by unit vector $\vec{n}_{1}$ and relative rotate about this axis. Intensity of this vector rotator expressed by generalized coordinate $\varphi_{2}$, angle of self rotation of heavy disk, taking into account first integral (29) of the differential equation (26) obtain the following form:

$$
\left|\overrightarrow{\mathrm{R}}_{012}\right|=2 \omega_{1} \sqrt{\dot{\varphi}_{02}^{2}+2 \Omega^{2}\left(\lambda \cos \varphi_{2}-\frac{1}{2} \cos ^{2} \varphi_{2}+\psi \sin \varphi_{2}\right)-2 \Omega^{2}\left(\lambda \cos \varphi_{02}-\frac{1}{2} \cos ^{2} \varphi_{02}+\psi \sin \varphi_{02}\right)}
$$

Intensity $R_{022}$ of two of these vector rotators, $\vec{R}_{022}$ and $\vec{R}_{2}$, from second set, depends on angular velocity $\omega_{2}$ and angular acceleration $\dot{\omega}_{2}$. For the considered system of the heavy gyro-rotor-disk dynamics, for obtaining expressions of intensities of vector rotators, $\vec{R}_{022}$ and $\vec{R}_{2}$, from second set, in the function of the generalized coordinate $\varphi_{2}$, angle of self rotation of heavy disk self rotation, we take into account a first integral (14) of nonlinear differential equation (11), and by using these result and previous expressions (15) of vector rotator we can write:

*intensities of the vectors rotators, $\vec{R}_{022}$ and $\vec{R}_{2}$, connected for the pole $O_{2}$ and rotate around self rotation axis, in the following form:

$$
\begin{aligned}
& \left|\vec{R}_{022}\right|=\left|\vec{R}_{022}\left(\varphi_{2}\right)\right|= \\
& =\Omega^{2} \sqrt{\left[-\left(\lambda-\cos \varphi_{2}\right) \sin \varphi_{2}+\psi \cos \varphi_{2}\right]^{2}+\left[\dot{\varphi}_{02}^{2}+2 \Omega^{2}\left(\lambda \cos \varphi_{2}-\frac{1}{2} \cos ^{2} \varphi_{2}+\psi \sin \varphi_{2}\right)-2 \Omega^{2}\left(\lambda \cos \varphi_{02}-\frac{1}{2} \cos ^{2} \varphi_{02}+\psi \sin \varphi_{02}\right)\right]^{2}}
\end{aligned}
$$
vector forms:

*vector rotators orthogonal to the self rotation axes are in the following

$$
\begin{aligned}
& \overrightarrow{\mathrm{R}}_{022}\left(\varphi_{2}\right)=\Omega^{2}\left[-\left(\lambda-\cos \varphi_{2}\right) \sin \varphi_{2}+\psi \cos \varphi_{2}\right] \frac{\left[\vec{n}_{2}, \vec{\rho}_{C}\right]}{\left[\vec{n}_{2}, \vec{\rho}_{C}\right]}+ \\
& +\Omega^{2}\left[\dot{\varphi}_{02}^{2}+2 \Omega^{2}\left(\lambda \cos \varphi_{2}-\frac{1}{2} \cos ^{2} \varphi_{2}+\psi \sin \varphi_{2}\right)-2 \Omega^{2}\left(\lambda \cos \varphi_{02}-\frac{1}{2} \cos ^{2} \varphi_{02}+\psi \sin \varphi_{02}\right)\right]\left[\frac{\vec{n}_{2},\left[\vec{n}_{2}, \vec{\rho}_{C}\right]}{\left.\| \vec{n}_{2}, \vec{\rho}_{C}\right]}\right. \\
& \overrightarrow{\mathrm{R}}_{2}\left(\varphi_{2}\right)=\Omega^{2}\left[-\left(\lambda-\cos \varphi_{2}\right) \sin \varphi_{2}+\psi \cos \varphi_{2}\right] \frac{\overrightarrow{\mathrm{D}}_{\bar{n}_{2}}^{\left(O_{2}\right)}}{\left|\overrightarrow{\mathrm{D}}_{\bar{n}_{2}}^{\left(O_{2}\right)}\right|}+ \\
& +\Omega^{2}\left[\dot{\varphi}_{02}^{2}+2 \Omega^{2}\left(\lambda \cos \varphi_{2}-\frac{1}{2} \cos ^{2} \varphi_{2}+\psi \sin \varphi_{2}\right)-2 \Omega^{2}\left(\lambda \cos \varphi_{02}-\frac{1}{2} \cos ^{2} \varphi_{02}+\psi \sin \varphi_{02}\right)\right]\left[\vec{n}_{2}, \frac{\overrightarrow{\mathrm{D}}_{\vec{n}_{2}}^{\left(O_{2}\right)}}{\left|\overrightarrow{\mathrm{D}}_{\bar{n}_{2}}^{\left(O_{2}\right)}\right|}\right]
\end{aligned}
$$




\section{Kinetic Pressures to Shaft Bearings of Rigid Body Coupled Rotations around Two Orthogonal No Intersecting Axes and with One Degree of Freedom}

By use previous derived vector equations (1) and (2) and approach to obtaining vector expressions (8) and (9) for kinetic pressures, $\vec{F}_{A 1}$ and $\vec{F}_{B 1}$, to fixed shaft bearings of rigid body coupled rotations around two no intersecting orthogonal axes and for system with one degree of freedom, it is easy to obtain vector expressions for kinetic pressures $\vec{F}_{A 2}$ and $\vec{F}_{B 2}$ (including component reactions of the rigid body weight) to self rotation shaft bearings, $A_{2}$ and $B_{2}$, of rigid body coupled rotations around two orthogonal no intersecting axes and for considered particular example in the following form:

$$
\begin{aligned}
& \vec{F}_{A \vec{n}_{2}}=\left[\left|\vec{S}_{\vec{n}_{1}}^{(O)}\right|\left(\vec{R}_{1}, \vec{n}_{2}\right)+\left|\vec{S}_{\vec{n}_{2}}^{(O)}\right|\left(\vec{R}_{21}, \vec{n}_{2}\right)-\left(\vec{G}, \vec{n}_{2}\right)\right] \vec{n}_{2} \\
& \vec{F}_{B 2}=\frac{1}{2}\left\{| \vec { \mathrm { S } } _ { \vec { n } _ { 2 } } ^ { ( O _ { 2 } ) } | \left[\vec{n}_{2},\left[\overrightarrow{\mathrm{R}}_{2}, \vec{n}_{2} \rrbracket+\left|\overrightarrow{\mathrm{S}}_{\vec{n}_{1}}^{\left(O_{2}\right)}\right|\left[\vec{n}_{2},\left[\overrightarrow{\mathrm{R}}_{1}, \vec{n}_{2} \rrbracket+\left|\overrightarrow{\mathrm{S}}_{\vec{n}_{2}}^{\left(O_{2}\right)}\right|\left[\vec{n}_{2},\left[\overrightarrow{\mathrm{R}}_{21}, \vec{n}_{2} \rrbracket-\left[\overrightarrow{n_{2}},\left[\vec{G}, \vec{n}_{2} \rrbracket\right\}+\right.\right.\right.\right.\right.\right.\right.\right. \\
& +\frac{1}{2 \ell}\left\{\left|\overrightarrow{\mathrm{D}}_{\vec{n}_{2}}^{\left(O_{2}\right)}\right|\left[\overrightarrow{\mathrm{R}}_{02}^{*}, \vec{n}_{2}\right]+\left|\overrightarrow{\mathrm{D}}_{\vec{n}_{1}}^{\left(O_{2}\right)}\right|\left[\overrightarrow{\mathrm{R}}_{02}^{*}, \vec{n}_{2}\right]+\left[\vec{n}_{1}, \vec{n}_{2}\right] \dot{\omega}_{1}\left(\vec{n}_{1}, \overrightarrow{\mathrm{J}}_{\vec{n}_{1}}^{\left(O_{2}\right)}\right)-\left[\left[\vec{\rho}_{C}, \vec{G}\right], \vec{n}_{2}\right]\right\}+ \\
& +\frac{1}{2 \ell}\left[\left\{\left[\left[\vec{n}_{2}, \overrightarrow{\mathrm{j}}_{\vec{n}_{1}}^{\left(O_{2}\right)}\right]+\left[\vec{n}_{1}, \overrightarrow{\mathrm{j}}_{\vec{n}_{2}}^{\left(O_{2}\right)}\right]+\mathrm{J}^{\left(O_{2}\right)}\left[\vec{n}_{1}, \vec{n}_{2}\right]\right\}, \vec{n}_{2}\right], \vec{n}_{1}\right] \\
& \vec{F}_{A T 2}=\frac{1}{2}\left\{| \vec { S } _ { \vec { n } _ { 2 } } ^ { ( O _ { 2 } ) } | \left[\vec{n}_{2},\left[\vec{R}_{2}, \vec{n}_{2} \rrbracket+\left|\vec{S}_{\vec{n}_{1}}^{\left(O_{2}\right)}\right|\left[\vec{n}_{2},\left[\overrightarrow{\mathrm{R}}_{1}, \vec{n}_{2}\right]+\left|\overrightarrow{\mathrm{S}}_{\vec{n}_{2}}^{\left(O_{2}\right)}\right|\left[\vec{n}_{2},\left[\overrightarrow{\mathrm{R}}_{21}, \vec{n}_{2}\right]\right]-\left[\vec{n}_{2},\left[\vec{G}, \vec{n}_{2}\right]\right\}-\right.\right.\right.\right. \\
& -\frac{1}{2 \ell}\left\{\left|\overrightarrow{\mathrm{D}}_{\vec{n}_{2}}^{\left(O_{2}\right)}\right|\left[\overrightarrow{\mathrm{R}}_{02}^{*}, \vec{n}_{2}\right]+\left|\overrightarrow{\mathrm{D}}_{\vec{n}_{1}}^{\left(O_{2}\right)}\right|\left[\overrightarrow{\mathrm{R}}_{01}^{*}, \vec{n}_{2}\right]+\left[\vec{n}_{1}, \vec{n}_{2}\right] \dot{\omega}_{1}\left(\vec{n}_{1}, \overrightarrow{\mathrm{J}}_{\vec{n}_{1}}^{\left(O_{2}\right)}\right)-\left[\left[\vec{\rho}_{C}, \vec{G}\right], \vec{n}_{2}\right]\right\}- \\
& \left.-\frac{1}{2 \ell}\left[\left\{\left[\vec{n}_{2}, \overrightarrow{\mathrm{J}}_{\vec{n}_{1}}^{\left(O_{2}\right)}\right]+\left[\vec{n}_{1}, \overrightarrow{\mathrm{J}}_{\vec{n}_{21}}^{\left(O_{2}\right)}\right]+\mathrm{J}^{\left(O_{2}\right)}\left[\vec{n}_{1}, \vec{n}_{2}\right]\right\}, \vec{n}_{2}\right], \vec{n}_{1}\right]
\end{aligned}
$$

where $\mathrm{J}^{\left(\mathrm{O}_{2}\right)}$ is matrix of tensor of mass inertia moments for pole $\mathrm{O}_{2}$. Previous expressions contain member which correspond to the bearing reactions of the rotor proper weight. After taking into account mass inertia moment vector for inclined disk and disk position with eccentricity of mass body center, we can write in scalar form components of kinetic pressures, $\vec{F}_{A 2}$ and $\vec{F}_{B 2}$ (including component reactions of the rigid body weight) to on bearings, $A_{2}$ and $B_{2}$, of the self rotation axis in the following form:

$$
\begin{aligned}
& F_{A u 2}=\frac{1}{2} m\left(e \ddot{\varphi}_{2} \sin \beta-O_{1} O_{2} \omega_{1}^{2} \cos \varphi_{2}-e \omega_{1}^{2} \sin \beta \sin \varphi_{2} \cos \varphi_{2}\right)+\frac{1}{2} m g\left(1+\frac{e}{\ell} \cos \beta\right) \sin \varphi_{2}+ \\
& \left.+\frac{1}{2 \ell}\left(\left(J_{u}-J_{v}-J_{n}\right) \dot{\varphi}_{2} \omega_{1}+J_{v n} \omega_{1}^{2} \cos \varphi_{2}\right) \sin \varphi_{2}+J_{v n} \ddot{\varphi}_{2}\right)-m \frac{e}{\ell} O_{1} O_{2} \omega_{1}^{2} \cos \beta \cos \varphi_{2}
\end{aligned}
$$




$$
\begin{aligned}
& F_{A v 2}=\frac{1}{2} m\left(e \dot{\varphi}_{2}^{2} \sin \beta+O_{1} O_{2} \omega_{1}^{2} \sin \varphi_{2}+e \omega_{1}^{2} \sin \beta \sin \varphi_{2} \sin \varphi_{2}\right)+\frac{1}{2} m g\left(1-\frac{e}{\ell} \cos \beta\right) \cos \varphi_{12}- \\
& \left.\quad-\frac{1}{2 \ell}\left(\left(J_{u}-J_{v}+J_{n}\right) \omega_{1} \dot{\varphi}_{2}-J_{v n} \omega_{1}^{2} \cos \varphi_{2}\right) \cos \varphi_{2}+J_{v n} \dot{\varphi}_{2}^{2}\right)-m \frac{e}{\ell} O_{1} O_{2} \omega_{1}^{2} \cos \beta \sin \varphi_{2} \\
& F_{B u 2}=\frac{1}{2} m\left(e \ddot{\varphi}_{2} \sin \beta-O_{1} O_{2} \omega_{1}^{2} \cos \varphi_{2}-e \omega_{1}^{2} \sin \beta \sin \varphi_{2} \cos \varphi_{2}\right)+\frac{1}{2} m g\left(1-\frac{e}{\ell} \cos \beta\right) \sin \varphi_{2}- \\
& \left.-\frac{1}{2 \ell}\left(\left(J_{u}-J_{v}-J_{n}\right) \dot{\varphi}_{2} \omega_{1}+J_{v n} \omega_{1}^{2} \cos \varphi_{2}\right) \sin \varphi_{2}+J_{v n} \ddot{\varphi}_{2}\right)-m \frac{e}{\ell} O_{1} O_{2} \omega_{1}^{2} \cos \beta \cos \varphi_{12} \\
& F_{B v 2}=\frac{1}{2} m\left(e \dot{\varphi}_{2}^{2} \sin \beta+O_{1} O_{2} \omega_{1}^{2} \sin \varphi_{2}+e \omega_{1}^{2} \sin \beta \sin \varphi_{2} \sin \varphi_{2}\right)+\frac{1}{2} m g\left(1-\frac{e}{\ell} \cos \beta\right) \cos \varphi_{2}+ \\
& \left.+\frac{1}{2 \ell}\left(\left(J_{u}-J_{v}+J_{n}\right) \omega_{1} \dot{\varphi}_{2}-J_{v n} \omega_{1}^{2} \cos \varphi_{2}\right) \cos \varphi_{2}+J_{v n} \dot{\varphi}_{2}^{2}\right)-m \frac{e}{\ell} O_{1} O_{2} \omega_{1}^{2} \cos \beta \sin \varphi_{2}
\end{aligned}
$$

Previous obtained expressions (22)-(25) of the components of kinetic pressures, $\vec{F}_{A 2}$ and $\vec{F}_{B 2}$ (including component reactions of the rigid body weight) to bearings, $A_{2}$ and $B_{2}$, of the self rotation axis in scalar form, is possible present in the following vector form:

$$
\begin{aligned}
& \vec{F}_{B 2}^{k i n}=F_{B u 2}^{k i n} \vec{u}_{2}+F_{B v 2}^{k i n} \vec{v}_{2}=\left(\frac{1}{2} m e \sin \beta-\frac{1}{2 \ell} J_{v n}\right)\left(\ddot{\varphi}_{u_{2}}+\dot{\varphi}^{2} \vec{v}_{2}\right)+ \\
& +\frac{1}{2 \ell}\left(J_{v n} \omega_{1} \cos \varphi_{2}-J_{n} \dot{\varphi}_{2}\right) \omega_{1}\left(\sin \varphi_{2} \vec{u}+\cos \varphi_{2} \vec{v}\right)- \\
& -\frac{1}{2} m \omega_{1}^{2}\left(a+e \sin \beta \cos \varphi_{2}+2 e \frac{a}{\ell} \cos \beta\right)\left(\cos \varphi_{2} \vec{u}-\sin \varphi_{2} \vec{v}\right)+ \\
& +\frac{1}{2 \ell}\left(J_{u}-J_{v}\right) \omega_{1} \dot{\varphi}_{2}\left(\sin \varphi_{2} \vec{u}-\cos \varphi_{2} \vec{v}\right) \\
& \vec{F}_{A 2}^{k i n}=F_{A n 2}^{k i n} \vec{u}_{2}+F_{A v 2}^{k i n} \vec{v}_{2}=\left(\frac{1}{2} m e \sin \beta+\frac{1}{2 \ell} J_{v n}\right)\left(\ddot{\varphi}_{2} \vec{u}_{2}+\dot{\varphi}_{2}^{2} \vec{v}_{2}\right)+ \\
& +\frac{1}{2 \ell}\left(J_{v n} \omega_{2} \cos \varphi_{2}+J_{n} \dot{\varphi}_{2}\right) \omega_{1}\left(\sin \varphi_{2} \vec{u}+\cos \varphi_{2} \vec{v}\right)- \\
& -\frac{1}{2} m \omega_{1}^{2}\left(a+e \sin \beta \cos \varphi_{2}-2 e \frac{a}{\ell} \cos \beta\right)\left(\cos \varphi_{2} \vec{u}-\sin \varphi_{2} \vec{v}\right)- \\
& -\frac{1}{2 \ell}\left(J_{u}-J_{v}\right) \omega_{1} \dot{\varphi}_{2}\left(\sin \varphi_{2} \vec{u}-\cos \varphi_{2} \vec{v}\right)
\end{aligned}
$$

Previous scalar expressions are suitable for analysis on the basis decompositions to the separate components with specific properties of intensity, directions, influence of some mass and geometrical properties and structure parameters, as well as angular velocities and other kinetic parameters of considered special example. 
By introducing the following unit vectors $\vec{w}_{1}, \vec{w}_{2}$ and $\vec{w}_{3}$

$\vec{w}_{1}=\vec{u}_{2} \sin \varphi_{2}+\vec{v}_{2} \cos \varphi_{2}, \vec{w}_{2}=\vec{u}_{2} \sin \varphi_{2}-\vec{v}_{2} \cos \varphi_{2}, \vec{w}_{3}=-\vec{u}_{2} \cos \varphi_{2}+\vec{v}_{2} \sin \varphi_{2}$

pressures $\vec{F}_{A g 2}$ and $\vec{F}_{B g 2}$ (reactions of the rigid body weight) to bearings $A_{2}$ and $B_{2}$, of the self rotation axis is possible to express in the following vector form:

$$
\begin{aligned}
& \vec{F}_{A g 2}=F_{A u g 2} \vec{u}_{2}+F_{A g v 2} \vec{v}_{2}=\frac{1}{2} m g\left(1+\frac{e}{\ell} \cos \beta\right) \sin \varphi_{2} \vec{u}_{2}+\frac{1}{2} m g\left(1-\frac{e}{\ell} \cos \beta\right) \cos \varphi_{2} \vec{v}_{2} \\
& \vec{F}_{B g 2}=F_{B g u 2} \vec{u}_{2}+F_{B g v 2} \vec{v}_{2}=\frac{1}{2} m g\left(1-\frac{e}{\ell} \cos \beta\right) \sin \varphi_{2} \vec{u}_{2}+\frac{1}{2} m g\left(1-\frac{e}{\ell} \cos \beta\right) \cos \varphi_{2} \vec{v}_{2}
\end{aligned}
$$

From last forms of the pressures, $\vec{F}_{\mathrm{Ag} 2}$ and $\vec{F}_{\mathrm{Bg} 2}$ (reactions of the rigid body weight) to bearings, $A_{2}$ and $B_{2}$, of the self rotation axis, we can see that is possible to separate component with same intensity, and opposite directions, and also component with same angular velocity in one or in other directions.

In Figure 3 some of the introduced unit vectors $\vec{u}_{2}, \vec{v}_{2}, \vec{w}_{1}, \vec{w}_{2}$ and $\vec{w}_{3}$ for analysis kinetic pressures $\vec{F}_{B 2}$ (including component reactions of the rigid body weight) to bearings, $A_{2}$ and $B_{2}$, of the self rotation axis used in expressions (28) schematically are presented with corresponding angular velocity and directions of rotations.
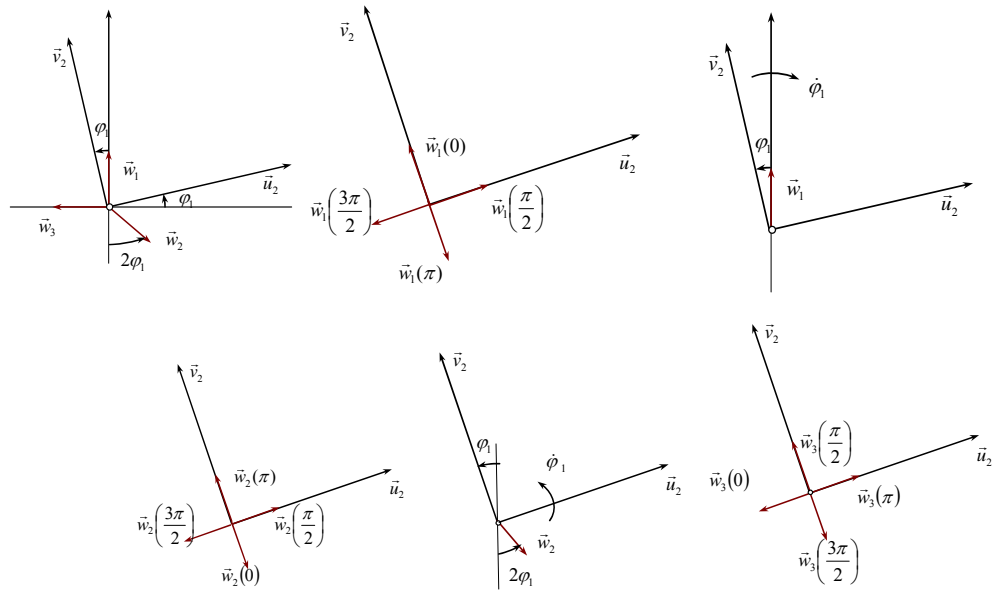

Figure 3

Schematically presentation of the unit vectors $\vec{u}_{2}, \vec{v}_{2}, \vec{w}_{1}, \vec{w}_{2}$ and $\vec{w}_{3}$, and their geometrical and kinematical relations with corresponding angular velocity and directions of rotations 
Components $F_{B 21}^{k i n}, F_{B 22}^{k i n}, F_{B 23}^{k i n}$ and $F_{B 24}^{k i n}$ of pure kinetic pressure $\vec{F}_{B 2}^{k i n}$ to bearing $B_{2}$, of the self rotation axis are in the following forms:

$$
\begin{aligned}
& F_{B 21}^{k i n}=\frac{1}{2 \ell}\left(J_{v n} \omega_{1} \cos \varphi_{2}-J_{n} \dot{\varphi}_{2}\right) \omega_{1}, \quad F_{B 22}^{k i n}=\frac{1}{2 \ell}\left(J_{u}-J_{v}\right) \omega_{1} \dot{\varphi}_{2} \\
& F_{B 23}^{k i n}=\frac{1}{2} m \omega_{1}^{2}\left(a+e \sin \beta \cos \varphi_{2}+2 e \frac{a}{\ell} \cos \beta\right), \quad F_{B 24}^{k i n}=\frac{1}{2} m e \sin \beta-\frac{1}{2 \ell} J_{v n}
\end{aligned}
$$

From previous expressions for components $F_{B 21}^{k i n}, F_{B 22}^{k i n}, F_{B 23}^{k i n}$ and $F_{B 24}^{k i n}$ of pure kinetic pressure $\vec{F}_{B 2}^{k i n}$ to bearing $B_{2}$, of the self rotation axis, we can conclude, that influence of disk position eccentricity is stronger to the components $F_{B 23}^{k i n}$ of pure kinetic pressure $\vec{F}_{B 2}^{k i n}$, and that intensity of component $F_{B 22}^{k i n}$ increase, and intensity of the component $F_{B 21}^{k i n}$ decrease with increasing of disk eccentricity. Intensity of the pure kinetic pressure $\vec{F}_{B 2}^{k i n}$ increase with increasing of disk eccentricity.

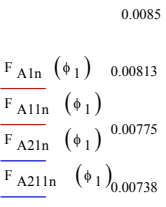

$\mathrm{a}^{*}$

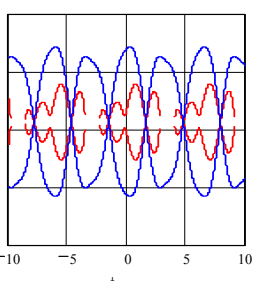

$\phi_{1}$

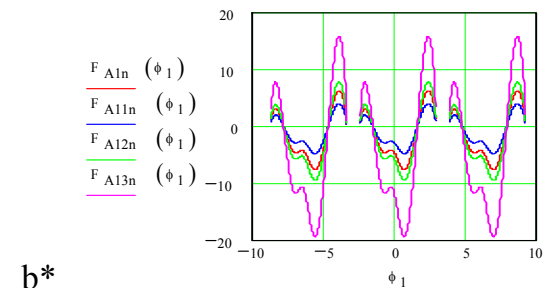

Figure 4

Intensity transformation of kinetic pressure component $F_{A 2 n 2}^{k i n}$ to self rotation shaft spherical bearing $A_{2}$ of rigid body coupled rotations around two orthogonal no intersecting axes and for system with one degree of freedom, in direction of the self rotation shaft axis for different disk eccentricity

\section{Graphical Presentation of Kinetic Pressures to Self Rotation Shaft Bearings of Rigid Body Coupled Rotations}

By use previous listed expressions as well as other no listed heir, and MathCad as a software tool, a numerical experiment was followed for analysis properties of the kinetic pressures and their corresponding components to the both shaft bearings. Selected graphical presentation is done in the Figures 4-10. All graphical 
presentation are obtained by analytical expressions derived in previous chapters of this paper.

In Figure $4 a^{*}$ and $b$ ( graphical presentation of intensity transformation of kinetic pressure component $F_{A 2 n 2}^{k i n}$ to self rotation shaft spherical bearing $A_{2}$ of rigid body coupled rotations around two orthogonal no intersecting axes and for system with one degree of freedom, in direction of the self rotation shaft axis and in function of self rotation relative angle $\varphi_{2}$, for different disk eccentricity, is presented.

In Figure 5 graphical presentation of intensity transformation of kinetic pressure component $F_{\mathrm{A} 2 \mathrm{~N} 2}^{k i n}$ to self rotation shaft spherical bearing $A_{2}$ of rigid body coupled rotations around two orthogonal no intersecting axes and for system with one degree of freedom, in orthogonal direction to the self rotation shaft axis, in function of self rotation relative angle $\varphi_{2}$, for different disk eccentricity, is presented.

$\mathrm{a}^{*}$

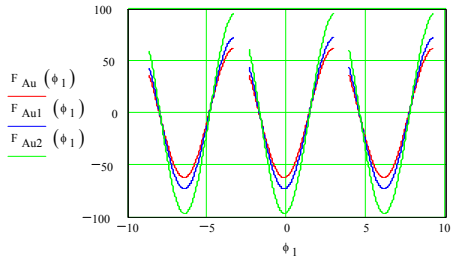

$b^{*}$

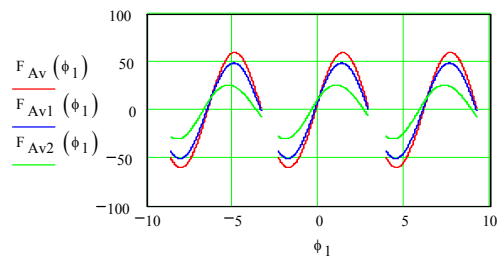

Figure 5

$\mathrm{a}^{*}$ and $\mathrm{b} *$ Intensity of kinetic pressure component $F_{A 2 N 2}^{k i n}$ to self rotation shaft spherical bearing $A_{2}$ of rigid body coupled rotations around two orthogonal no intersecting axes and for system with one degree of freedom, in orthogonal direction to the self rotation shaft axis, for different value of disk eccentricity

In Figure $6\left(\mathrm{a}^{*}\right),\left(\mathrm{c}^{*}\right)$ and $\left(\mathrm{d}^{*}\right)$ the intensity of kinetic pressure component of $F_{B 2 \mathrm{~N} 2}^{k i n}$ to self rotation cylindrical bearing $B_{2}$ of rigid body coupled rotations around two orthogonal no intersecting axes in direction of $\vec{R}_{2}$ and for system with one degree of freedom, in orthogonal direction to the self rotation shaft axis, for different value of disk angle $\beta$ skew position is presented. In Figure $8\left(b^{*}\right)$ Intensity of the vector rotator $\vec{R}_{2}$ in function of the value of disk angle $\beta$ skew positions is presented.

In Figure 7 graphical presentation of intensity transformation of kinetic pressure component $F_{B 2 N 2}^{k i n}$ to self rotation shaft cylindrical bearing $B_{2}$ of rigid body coupled rotations around two orthogonal no intersecting axes and for system with one degree of freedom, in orthogonal direction to the self rotation shaft axis, in function of self rotation relative angle $\varphi_{2}$, for different disk eccentricity, is presented. 


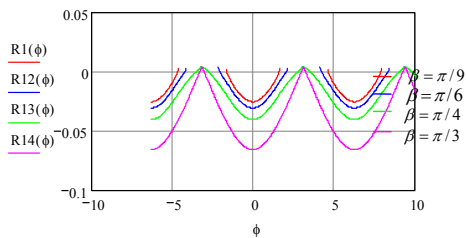

$a^{*}$

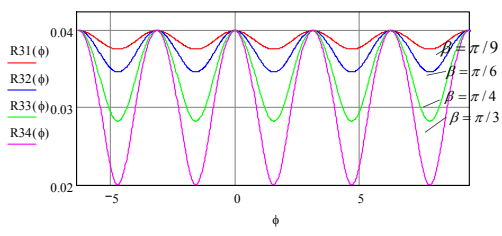

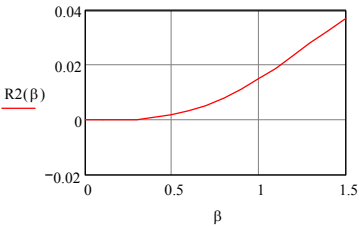

$b^{*}$

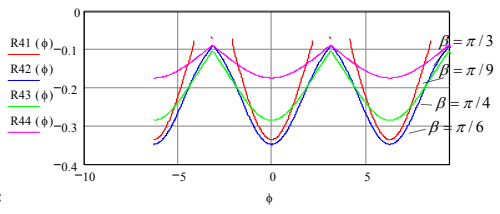

$d^{*}$

Figure 6

$\left(\mathrm{a}^{*}\right),\left(\mathrm{c}^{*}\right)$ and $\left(\mathrm{d}^{*}\right)$ Intensity of kinetic pressure component of $F_{B 2 N 2}^{\text {kin }}$ to self rotation cylindrical bearing $B_{2}$ of rigid body coupled rotations around two orthogonal no intersecting axes in direction of $\vec{R}_{2}$ and for system with one degree of freedom, in orthogonal direction to the self rotation shaft axis, for different value of disk angle $\beta$ skew position..( $\mathrm{b}^{*}$ ) Intensity of the vector rotator $\overrightarrow{\mathrm{R}}_{2}$ in function of the value of disk angle $\beta$ skew position

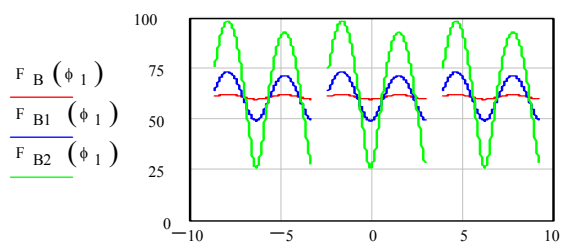

Figure 7

Intensity of kinetic pressure component $F_{B 2 N 2}^{k i n}$ to self rotation shaft cylindrical bearing $B_{2}$ of rigid body coupled rotations around two orthogonal no intersecting axes and for system with one degree of freedom, in orthogonal direction to the self rotation shaft axis, for different value of disk eccentricity

In Figure 8 graphical presentation of intensity transformation of kinetic pressure component $F_{B 2 N 2}^{k i n}$ to self rotation shaft cylindrical bearing $B_{2}$ of rigid body coupled rotations around two orthogonal no intersecting axes and for system with one degree of freedom, in orthogonal direction to the self rotation shaft axis, in function of self rotation relative angle $\varphi_{2}$, for different disk eccentricity, is presented. In Figure 9 intensities of kinetic pressure deviation couple to self rotation shaft bearings of rigid body coupled rotations around two orthogonal no intersecting axes and for system with one degree of freedom, in orthogonal direction to the self rotation shaft axis, for different value of disk eccentricity are presented. 

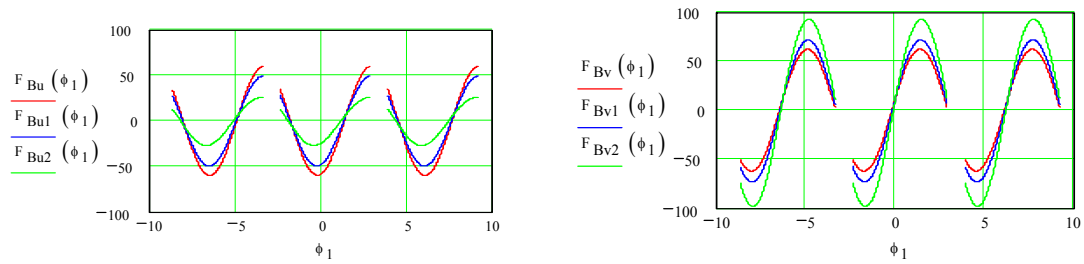

Figure 8

Intensity of kinetic pressure component $F_{B 2 N 2}^{\text {kin }}$ to self rotation shaft cylindrical bearing $B_{2}$ of rigid body coupled rotations around two orthogonal axes without intersection and for system with one degree of freedom, in orthogonal direction to the self rotation shaft axis, for different value of disk eccentricity.

$a^{*}$

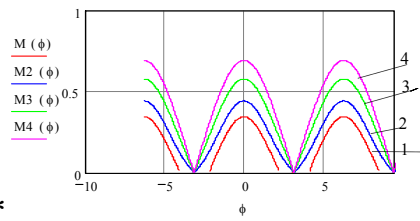

$b^{*}$

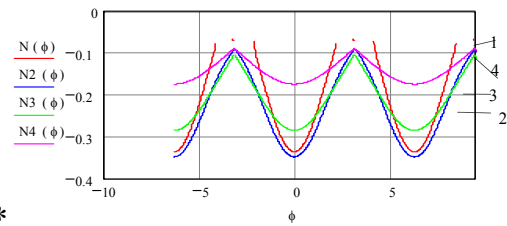

Figure 9

Intensity of kinetic pressure deviation couple to self rotation shaft bearings of rigid body coupled rotations around two orthogonal no intersecting axes and for system with one degree of freedom, in orthogonal direction to the self rotation shaft axis, for different value of disk eccentricity.

\section{Concluding remarks}

Complexity of the single rigid body motion with coupled rotations about no intersecting axes by vector method based on the mass moment vectors and vector rotators coupled for pole on selfrotation axis and component angular velocity axes is presented by sampler vector expressions them usually scalar forms in professional books in this area. New approach and new composition of this vector method open new way for applications to the multi-body system dynamics with coupled multi-rotations about nonintersecting axes. New vector expressions for linear momentum and angular momentum and their derivatives of the single rigid body complex motion by coupled rotations about nonintersecting axes expressed by new introduced mass moments vectors and their very elegant form open new possibility for generalizations these expressions for describing multi rigid body system complex motion by coupled multi-rotations about higher numbers of nonintersecting axes large present in many real mechanical engineering systems and robotic system dynamics with coupled multi-rotations.

\section{Acknowledgement}

Parts of this research were supported by the Ministry of Sciences and Technology of Republic of Serbia through Mathematical Institute SANU Belgrade Grant ON174001 Dynamics of hybrid systems with complex structures. Mechanics of materials and Faculty of Mechanical Engineering University of Niš and Faculty of Mechanical Engineering University of Kragujevac. 


\section{References}

[1] Andonov, A. A., Vitt, A. A., Haykin, S. E., (1981), Teoriya kolebaniy, Nauka, Moskva, p. 568

[2] Hedrih (Stevanović) K., (1992), On Some Interpretations of the Rigid Bodies Kinetic Parameters, XVIIIth ICTAM HAIFA, Apstracts, pp. 73-74

[3] Hedrih (Stevanović) K., (1993), Same Vectorial Interpretations of the Kinetic Parameters of Solid Material Lines, ZAMM. Angew.Math. Mech. 73(1993) 45, T153-T156

[4] Hedrih (Stevanović) K., (1993), The Mass Moment Vectors at n-dimensional Coordinate System, Tensor, Japan, Vol 54 (1993), pp. 83-87

[5] Hedrih (Stevanović) K., (2001), Vector Method of the Heavy Rotor Kinetic Parameter Analysis and Nonlinear Dynamics, University of Niš 2001, Monograph, p. 252 (in English), YU ISBN 86-7181-046-1

[6] Hedrih (Stevanović) K., (1998), Vectors of the Body Mass Moments, Monograph paper, Topics from Mathematics and Mechanics, Mathematical institute SANU, Belgrade, Zbornik radova 8(16), 1998, pp. 45-104, Published in 1999 (in English), (Zentralblatt Review)

[7] Hedrih (Stevanović) K., (1998), Derivatives of the Mass Moments Vectors with Applications, Invited Lecture, Proceedings, $5^{\text {th }}$ National Congress on Mechanics, Ioannina, 1998, pp. 694-705

[8] Hedrih (Stevanović) K., (1994), Interpretation of the Motion of a Heavy Body around a Stationary Axis in the Field with Turbulent Damping and Kinetic Pressures on Bearing by Means of the Mass Moment Vector for the Pole and the Axis, Facta Universitatis Series Mechanics, Automatic Control and Robotics, Vol. 1, No. 4, 1994, pp. 519-538

[9] Hedrih (Stevanović) K., (1998), Vector Method of the Kinetic Parameters Analysis of the Rotor with Many Axes and Nonlinear Dynamics, Parallel General Lecture, Third International Conference on Nonlinear Mechanics (ICNM III), August 17-20, 1998, Shanghai, China, pp. 42-47

[10] Hedrih (Stevanović) K., (2004), Contribution to the Coupled Rotor Nonlinear Dynamics, Advances in nonlinear Sciences, Monograph, Belgrade, Academy of Nonlinear Sciences, 229-259. ISBN 86-905633-0-X UDC 530-18299(082) 51-73:53(082) UKUP. STR. 261

[11] Hedrih (Stevanović) K., Interpretation of the Motion Equations of a Variable Mass Object Rotating around a Atationary Axis by Means of the Mass Moment Vector for the Pole and the Axis, Procedings of the $4^{\text {th }}$ Greek National Congress on Mechanics, Vol. 1, Mechanics of Solids, Democritus University of Trace, Xanthi, 1995, pp. 690-696

[12] Hedrih (Stevanovic) K. and Veljovic Lj., Vector Rotators of Rigid Body Dynamics with Coupled Rotations around Axes without Intersection, Hindawi Publishing Corporation, Mathematical Problems in Engineering, Volume 2011, Article ID 351269, 26 pages, doi:10.1155/2011/351269 